\title{
Initial experiences with preoperative three-dimensional image reconstruction technology in laparoscopic pyeloplasty for ureteropelvic junction obstruction
}

\author{
Weijie Zhu ${ }^{1 \#}$, Shengwei Xiong ${ }^{1 \#}$, Chunru Xu${ }^{1 \#}$, Zhenpeng Zhu ${ }^{1}$, Zhihua Li $^{1}$, Lei Zhang ${ }^{1}$, Hua Guan ${ }^{1}$, \\ Yanbo Huang ${ }^{1}$, Peng Zhang ${ }^{2}$, Hongjian Zhu' ${ }^{3}$, Jian Lin ${ }^{1}$, Xuesong Li ${ }^{1}$, Liqun Zhou ${ }^{1}$ \\ ${ }^{1}$ Department of Urology, Peking University First Hospital, Institute of Urology, Peking University, National Urological Cancer Centre, Beijing, \\ China; ${ }^{2}$ Department of Urology, Emergency General Hospital, Beijing, China; ${ }^{3}$ Department of Urology, Beijing Jiangong Hospital, Beijing, China \\ Contributions: (I) Conception and design: W Zhu, J Lin, X Li; (II) Administrative support: L Zhou, S Xiong, C Xu; (III) Provision of study materials \\ or patients: Z Li, Z Zhu; (IV) Collection and assembly of data: H Guan, Y Huang; (V) Data analysis and interpretation: P Zhang, H Zhu; (VI) \\ Manuscript writing: All authors; (VII) Final approval of manuscript: All authors. \\ \#These authors contributed equally to this work. \\ Correspondence to: Jian Lin; Xuesong Li. Department of Urology, Peking University First Hospital, Institute of Urology, Peking University, National \\ Urological Cancer Centre, No. 8 Xishiku St., Xicheng District, Beijing 100034, China. Email: pineneedle@sina.com.
}

Background: To explore the clinical value of three-dimensional image reconstruction technology (3DIT) on preoperative surgical planning and perioperative outcomes in laparoscopic pyeloplasty (LP).

Methods: Data of 25 patients with ureteropelvic junction obstruction (UPJO) admitted to our hospital from January 2018 to January 2019 was analyzed retrospectively. All patients underwent preoperative enhanced computed tomography (CT) scanning. In the 12 cases in the 3DIT group, preoperative planning involved the use of virtual operation and morphometry based on reconstruction of the CT data into threedimensional (3D) images. Surgery in the other 13 cases was performed with traditional CT examination. Demographic, surgical outcome, and postoperative parameters were compared between these two groups.

Results: Reconstructed 3D images clearly showed the spatial structural relationships between the UPJO and surrounding blood vessels. In all 25 cases surgery was completed with no conversion to open surgery. Preoperative 3DIT analyses resulted in significant improvements to mean operation time (107.76 vs. 141.58 min, $\mathrm{P}=0.024)$, mean time of dissociating ureteropelvic junction (UPJ) (11.26 vs. $19.40 \mathrm{~min}, \mathrm{P}=0.020)$, and mean estimated blood loss volume $(23.84$ vs. $49.16 \mathrm{~mL}, \mathrm{P}=0.028)$. There were no statistically significant differences in perioperative complications, postoperative hospital stays or postoperative drainage time.

Conclusions: 3DIT based on enhanced CT scans is of clinical value in the treatment of UPJO, as it can provide accurate anatomical information and reliable guidance for preoperative operation planning, and it facilitates image-guided LP.

Keywords: Three-dimensional image reconstruction technology (3DIT); surgical plan; laparoscopic pyeloplasty; ureteropelvic junction dissection

Submitted Jul 04, 2021. Accepted for publication Sep 28, 2021.

doi: $10.21037 /$ tau-21-590

View this article at: https://dx.doi.org/10.21037/tau-21-590

^ ORCID: 0000-0003-2699-8377. 


\section{Introduction}

Ureteropelvic junction obstruction (UPJO) is a urinary tract obstruction disease that is characterized by blockage or obstruction of urine flow from the renal pelvis into the proximal upper ureter (1). The overall incidence of UPJO is approximately 67 cases per 100,000 adults per year, and it is considered to be a common cause of hydronephrosis (2). The etiologic factors of UPJO can be categorized as congenital and acquired, which can be further divided into intrinsic and extrinsic. Intrinsic factors of UPJO include congenital impaired ureteric peristalsis, ureteric kinking and scarring of the ureteric valve, urothelial neoplasm and acquired renal calculi. Extrinsic factors include congenital crossing of the lower pole vessels, horseshoe kidney and duplex kidney, acquired iatrogenic strictures and fibroepithelial polyps (3-5). Crossing vessels that distort the configuration of the ureteropelvic junction (UPJ) are often regarded as the main extrinsic cause of UPJO (6-8). These vessels include aberrant, accessory, and early branching renal arteries and veins.

Without early and effective treatment, UPJO can cause hydronephrosis, leading to interstitial fibrosis and nephron loss and can eventually lead to renal failure. Therefore, it is important to take timely and appropriate measures. In recent decades, surgical techniques for management of UPJO have made great progress. In the 1990s, open approaches began to be replaced by laparoscopic AndersonHynes pyeloplasty and then endopyelotomy, and robotassisted approaches were introduced in $2002(9,10)$.

A reflection of this trend is the development of laparoscopic pyeloplasty (LP), which is now the preferred treatment method, as it is accompanied by improved intraoperative visualization, reduced blood loss, lower postoperative morbidity, faster recovery and better cosmetic effects (11). However, LP has been proven to be a challenging procedure with a steep learning curve for surgeons, who must have strong experience with laparoscopic procedures and must have advanced laparoscopic skills including the ability to perform intracorporeal suturing and knotting (12). Because of the challenges presented by this technique, accurate preoperative assessment is essential to improve surgical planning, decrease intraoperative surgical risk and difficulty, and to reduce the incidence of postoperative complications.

Accurate preoperative evaluation requires the utilization of modern imaging technology. Recently, a novel technology based on computed tomography (CT) or magnetic resonance imaging (MRI), three-dimensional image reconstruction (3DIT), has emerged as a useful tool for making a targeted region visible and tangible. The use of this technique allows simulated manipulations to be conducted to assess operative features preoperatively. In a similar way, previous studies have reported that helical CT with vascular reconstruction can assist the surgeon to observe the relationship between the UPJ and peripheral structures, to detect of crossing vessels before surgery and to perform virtual manipulation (13). To this point, however, no research has investigated the superiority of three-dimensional (3D) techniques over conventional twodimensional (2D) CT as applied to LP procedures. Here, then, we provide a comparative evaluation of perioperative outcomes upon the application of 3DIT to LP. We present the following article in accordance with the STROBE reporting checklist (available at https://dx.doi.org/10.21037/ tau-21-590).

\section{Methods}

\section{Study population}

From January 2018 to January 2019, a total data of 25 patients from our hospital who were diagnosed with UPJO and who received standardized dismembered LP by a single experienced surgeon were analyzed retrospectively (14). Of this cohort, 12 patients underwent LP combined with three-dimensional visualization surgical planning (3DIT group), while 13 patients underwent LP under conventional two-dimensional CT assessment (non-3DIT group).

Patients were selected according to the following criteria: (I) anatomic abnormalities of the urinary tract as determined by preoperative CT; (II) functioning kidneys that responded appropriately to attempted ureter repairs; and (III) availability of follow-up data of at least 6 months. Patients with missing perioperative or postoperative data were excluded from the analysis.

Patients were classified according to their willingness to submit to the preoperative $3 \mathrm{D}$ visualization technique. All patients were informed of the treatment strategy and potential risks and complications. The study was conducted in accordance with the Declaration of Helsinki (as revised in 2013). The study was approved by the Ethics Committee of Peking University First Hospital (approval number: 2020-283) and individual consent for this 

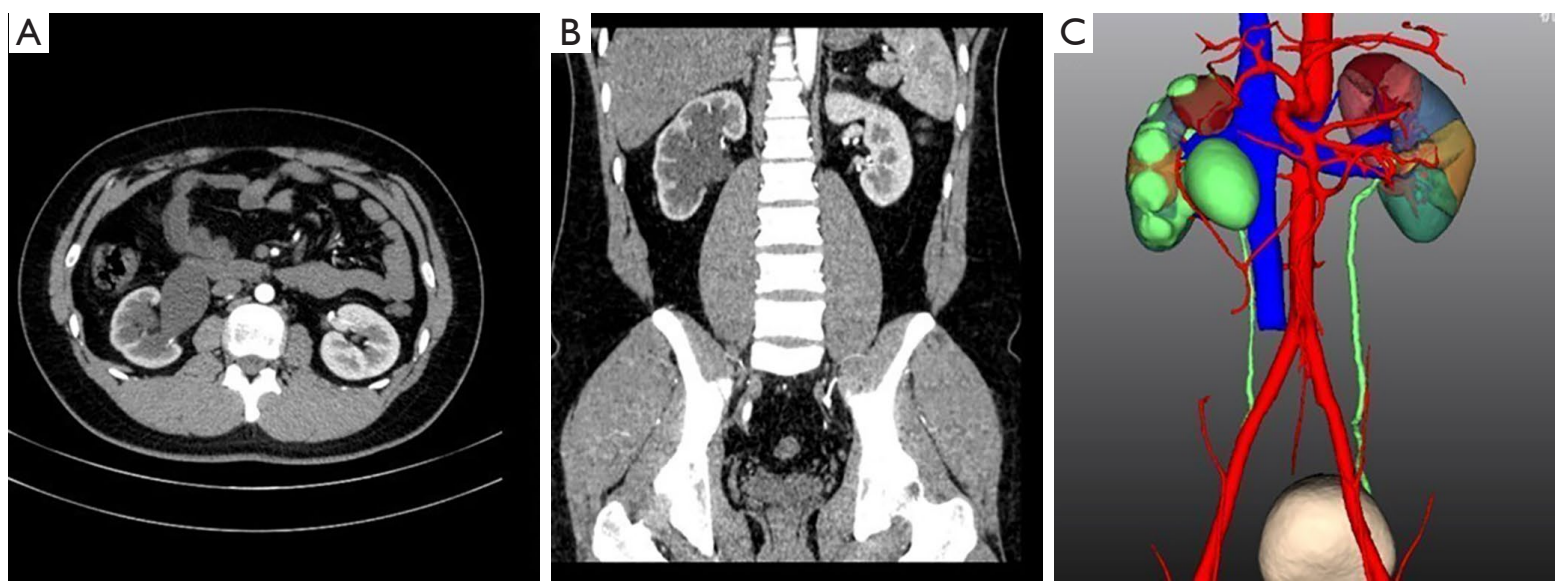

Figure 1 Three-dimensional (3D) imaging reconstruction model of urinary system based on computerized tomography (CT). (A) Bilateral kidneys and collecting systems shown by the transaxial CT scan; (B) bilateral kidneys and collecting systems shown by the coronary CT scan; (C) $3 \mathrm{D}$ imaging reconstruction model of urinary system and vessels.

retrospective analysis was waived.

\section{$3 D$ image reconstruction technology}

Enhanced CT scans were performed on all 25 patients using a 64-slice CT system with a slice thickness of 0.625 $\mathrm{mm}$ (Philips, Best, The Netherlands). The original CT data for subsequent analysis was set to the best image quality and stored in Digital Imaging and Communications in Medicine format. Different phases of the CT data were segmented and reconstructed to create $3 \mathrm{D}$ digital images with a Medical Imaging Three Divisional Visualization System (Yorktal, Inc., Shenzhen, Guangdong, China). A 3D model of the retroperitoneal anatomical structures, including renal parenchyma, upper urinary tract, renal vessels, abdominal aorta and inferior vena cava, was reconstructed simultaneously using the region-growing method based on arterial and parenchymal phase data (Figure 1). Information regarding the collecting system was obtained based on secretory phase data (15).

\section{Morphometry and preoperative planning}

The reconstructed $3 \mathrm{D}$ model was observed by $360^{\circ}$ rotation along all axes. Each part of the reconstructed anatomical structure allowed the user to zoom in and zoom out at selected angles. The surgeon was able to rotate, amplify or delete areas or to make certain areas transparent in order to better visualize the anatomy of the involved surgical area. Important retroperitoneal structures were tinted with different colors for distinguishing prior to the surgery. This system created a virtual reality environment of the surgical field and allowed the surgeon to perform virtual manipulation on the screen. After the simulated surgery was performed, potential intraoperative events were predicted, which helped the surgeon to make appropriate surgical plans.

\section{Statistical analysis}

Standard variables were retrospectively collected from our Reconstruction of Urinary Tract: Technology, Epidemiology and Result (RECUTTER) database (https:// pkufh.yorktal.com). Data on age, gender, side of UPJO, and the presence of a crossing vessel were gathered for comparison between the groups. The efficiency of 3DIT was assessed by operating time and recorded as the total operating time, from skin incision to wound closure, and the UPJ dissection time, from the opening of Gerota's fascia to the completion of the exposure immediately before the pelvic incision.

Postoperative follow-up occurred at 3 and 6 months and then once per year. Follow-up examinations included clinical evaluation, serological examination of creatinine levels and estimated glomerular filtration rate, renal ultrasound examination, diuretic nephropathy, and CT imaging. The criteria for success were relief of subjective pain, improvement of hydronephrosis, and stabilization or improvement of renal function.

Statistical analysis was performed using SPSS software 
Table 1 Clinical data analysis of patients diagnosed with UPJO with ectopic vessels in 3DIT group

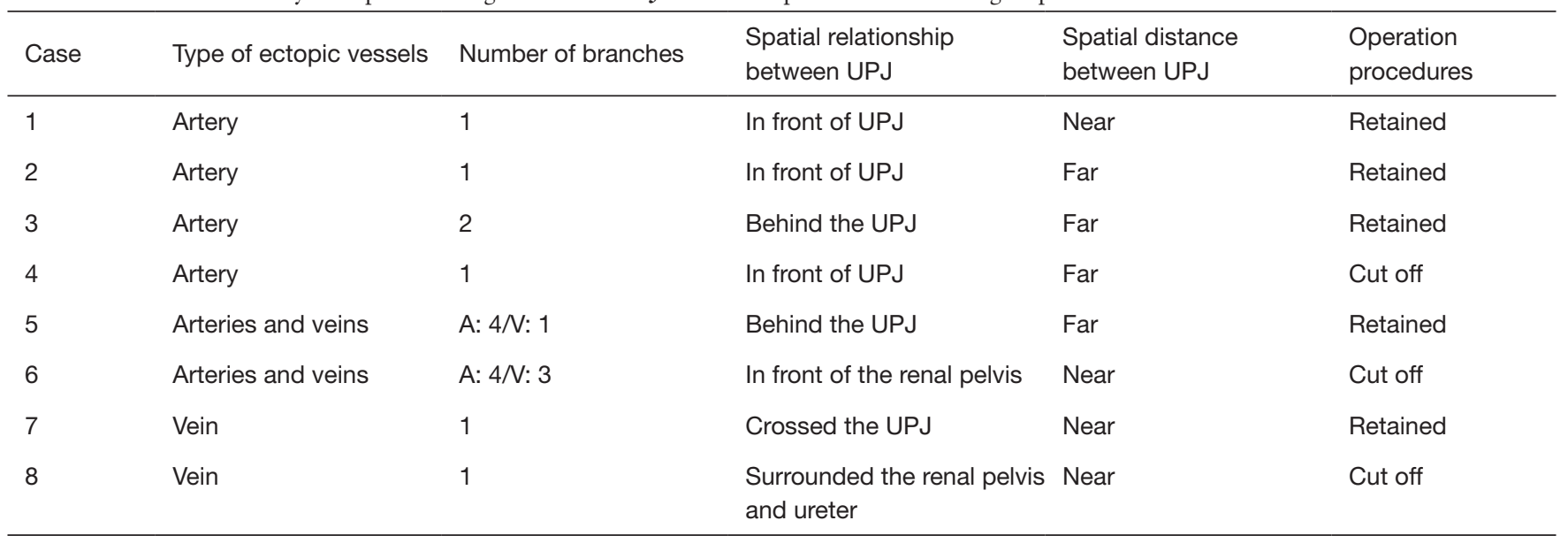

3DIT, three-dimensional images reconstruction technology; UPJ, ureteropelvic junction; UPJO, ureteropelvic junction obstruction; A, artery; $\mathrm{V}$, vein.
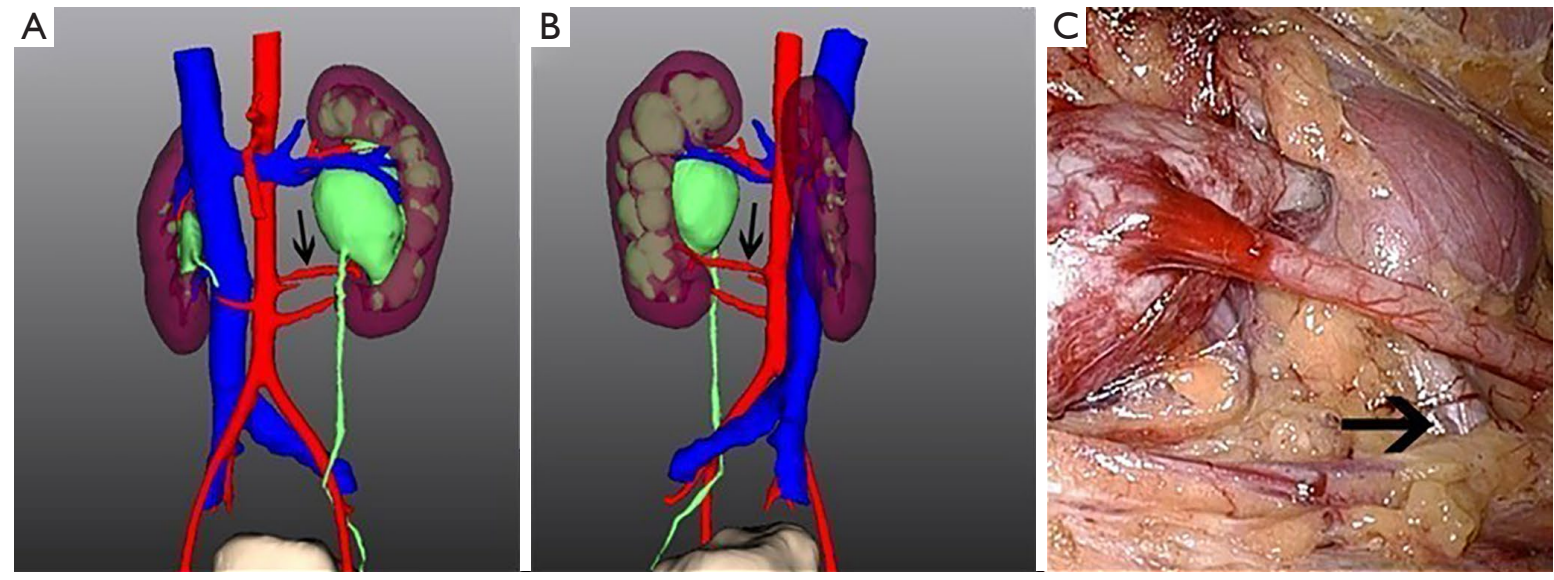

Figure 2 Ureteropelvic junction obstruction with left renal crossing artery (arrows). (A) Anterior aspect in three-dimensional (3D) imaging model; (B) posterior aspect in in 3D imaging model; (C) crossing artery located at the lower pole of the left kidney, behind the ureter, intraoperatively.

version 23.0 (SPSS, Inc., IBM Corp., Somers, NY, USA). Descriptive data are expressed in terms of frequency and percentage. Fisher exact tests or chi square tests were used to compare percentages between the two groups. Nonparametric two sample median tests were used to compare median values. Differences with bilateral $\mathrm{P}<0.05$ were considered statistically significant.

\section{Results}

\section{$3 D$ reconstruction of renal crossing vessels}

Of the 12 patients without preoperative $3 \mathrm{D}$ reconstruction,
5 were determined to have renal crossing vessels, but it should be noted that ordinary CT imaging does not allow for conclusive distinguishing of veins from arteries.

Of the 13 patients with preoperative $3 \mathrm{D}$ reconstruction, eight patients with UPJO in the 3DIT group showed the presence of ectopic blood vessels (Table 1). The ectopic blood vessels of 4 patients were arteries, including 3 ectopic arteries in front of the renal pelvis and ureter and 1 ectopic artery behind the renal pelvis and ureter (Figure 2). The ectopic blood vessels of 2 patients were both arteries and veins: in 1 case, the vessel was in front of the renal pelvis and ureters, and in the other case, the vessel was behind the 

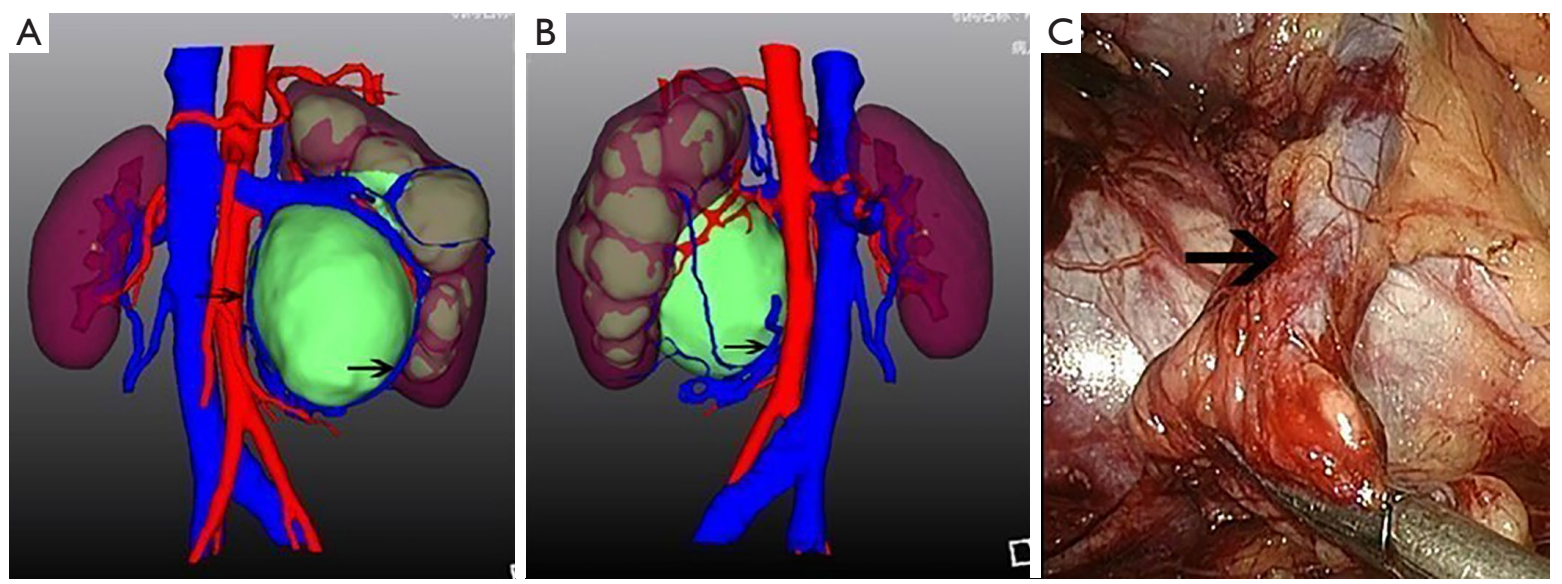

Figure 3 Ureteropelvic junction obstruction with left renal ectopic vein (arrows). (A) Anterior aspect in three-dimensional (3D) imaging model; (B) posterior aspect in 3D imaging model; (C) renal ectopic vein made a circuit around the left renal pelvis, intraoperatively.

Table 2 Preoperative characteristics of patient

\begin{tabular}{lccc}
\hline Parameter & Non-3D reconstruction & 3D reconstruction & P value \\
\hline No. of patients & 12 & 13 & $7 / 6$ \\
Gender (M/F) & $8 / 4$ & $27.30 \pm 14.70[8-69]$ & 0.533 \\
Age (year), mean \pm SD [range] & $33.33 \pm 15.08[18-73]$ & $21.4 \pm 3.5$ & 0.318 \\
BMI (kg/m ${ }^{2}$ ), mean \pm SD & $20.2 \pm 4.5$ & $8 / 5$ & 0.260 \\
Laterality (left/right) & $6 / 6$ & 5 & 0.800 \\
History of urological surgery & 4 & 8 & 0.420 \\
Crossing vessels & 5 & & 0.915 \\
Presentation & & 9 & 0.341 \\
Osphyalgia & 6 & 1 & \\
Hematuresis & 3 & 5 & \\
Vascular variation & 5 & & \\
\hline
\end{tabular}

3D, three-dimensional; No., number; M, male; F, female; BMI, body mass index; SD, standard deviation.

renal pelvis and ureters. Only 2 patients had ectopic blood veins. Here, in 1 case, the vein crossed the UPJ, while in the other case, the vein surrounded the renal pelvis and ureter (Figure 3).

\section{LP without preoperative $3 D$ reconstruction}

Preoperative characteristics of patients are presented in Table 2. Twelve patients underwent LP without preoperative 3D imaging reconstruction, including 7 males and 6 females. The mean age of the patients was $33.33 \pm 15.08$ years (range, $18-73$ years). Six patients had pyeloplasty performed on the left side, and six patients had pyeloplasty performed on the right side. Three patients visited the hospital complaining of hematuria, and six patients complained of osphyalgia. The mean surgery time, time of dissociating UPJ and blood loss during operation were $141.58 \pm 46.90$ min (range, $77-253 \mathrm{~min}$ ), $19.40 \pm 9.13 \mathrm{~min}$ (range, 7.9-41.5 min) and $49.16 \pm 36.54 \mathrm{~mL}$ (range, $20-160 \mathrm{~mL}$ ), respectively. There was no conversion to open surgery. The mean time of drain removal was $3.41 \pm 0.99$ days (range, $2-6$ days), and the average time of hospitalization was $4.16 \pm 2.55$ days (range, 3-12 days). Five patients experienced postoperative fever (Clavien-Dindo grade I). The mean postoperative follow- 
Table 3 Perioperative and postoperative data of patients

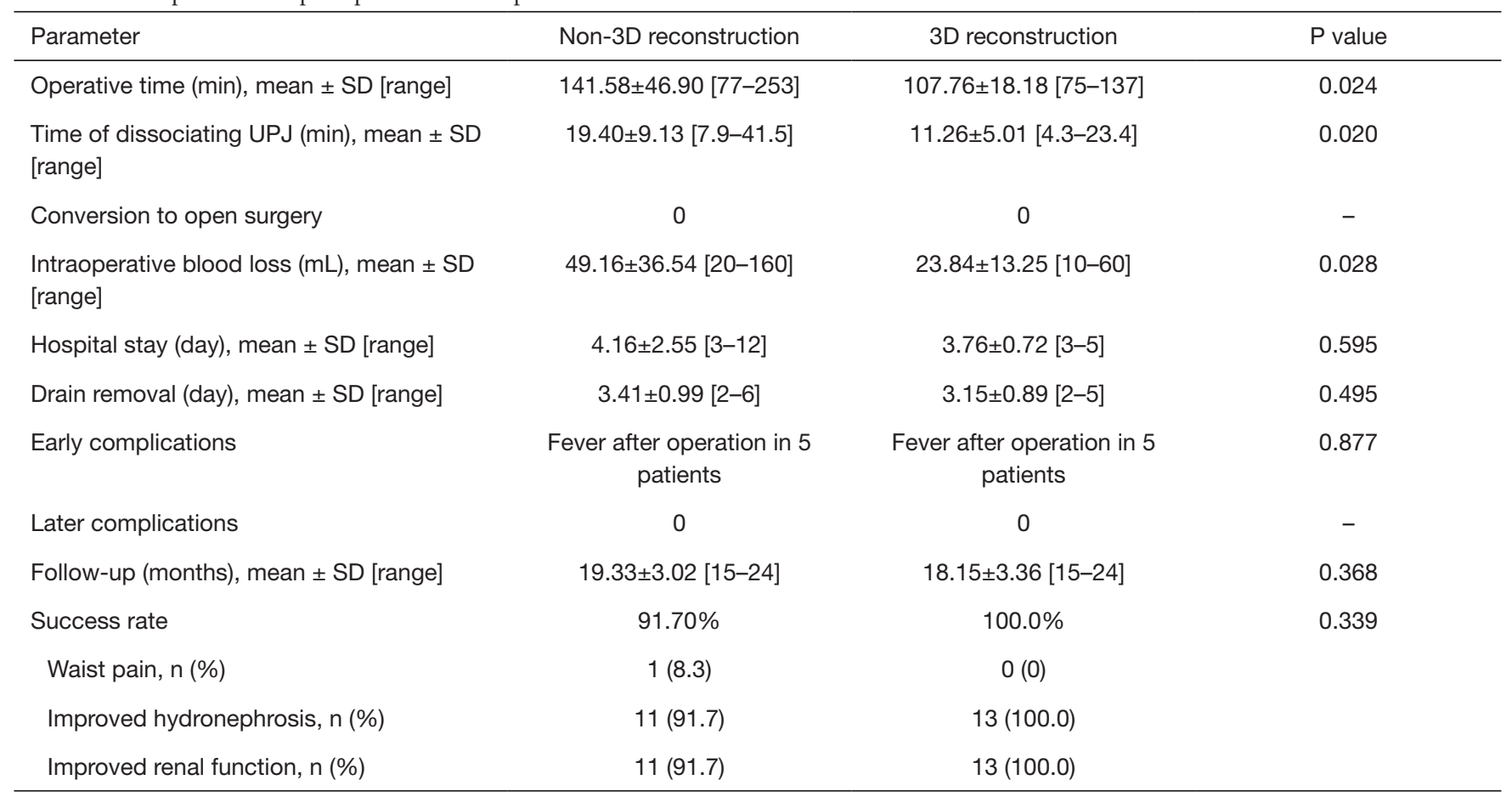

SD, standard deviation; UPJ, ureteropelvic junction.

up was 19.33 months (range, 15-24 months), and 11 of the 12 patients had reduced hydronephrosis and improved renal function.

\section{$L P$ with preoperative $3 D$ reconstruction}

$\mathrm{LP}$ with preoperative $3 \mathrm{D}$ imaging reconstruction was successfully performed in 13 cases, including 7 males and 6 females (Table 2). The mean age of patients was $27.30 \pm 14.70$ years (range, $8-69$ years). Eight patients received surgery on the left side, and five patients on the right side. Six patients were admitted to hospital with complaints of loin pain, and three patients were admitted with hematuresis. The mean operative time, time of dissociating UPJ and intraoperative blood loss were $107.76 \pm 18.18 \mathrm{~min}$ (range, $75-137 \mathrm{~min}$ ), 11.26 \pm 5.01 min (range, 4.3-23.4 min) and $23.84 \pm 13.25 \mathrm{~mL}$ (range, 10-60 mL), respectively. All patients were treated successfully without any conversion to open surgery. The mean time of drain removal was $3.15 \pm 0.89$ days (range, 2-5 days), and the mean hospitalization time was $3.76 \pm 0.72$ days (range, $3-5$ days). Five patients complained of postoperative fever (Clavien-Dindo grade I). The symptoms of preoperative discomfort disappeared, hydronephrosis decreased and the renal function was stabilized or improved at a mean follow-up of 18.15 months (range, 15-24 months).

As shown in Table 3, preoperative 3DIT analyses resulted in significant improvements to mean operation time (107.76 vs. $141.58 \mathrm{~min}, \mathrm{P}=0.024)$, mean time of dissociating UPJ (11.26 vs. $19.40 \mathrm{~min}, \mathrm{P}=0.020$ ), and mean estimated blood loss volume ( 23.84 vs. $49.16 \mathrm{~mL}, \mathrm{P}=0.028$ ). There were no statistically significant differences in perioperative complications, postoperative hospital stays or postoperative drainage time.

\section{Discussion}

Over the past two decades, minimally invasive treatment of UPJO has developed and is becoming increasingly popular. LP, in particular, has been used successfully to correct all possible causes of UPJ obstruction, including kinks or compression associated with crossing vessels or inherent narrowing of the UPJ (16). LP is a difficult surgical technique that demands superior surgeon expertise, including superior dexterity and intracorporeal suturing skills, and it especially challenges novice urological surgeons. Variance of both venous and arterial crossing 
vessels and the complexity of features of UPJO make exposure of the dilated pelvis and UPJO difficult (17).

The need to reduce the difficulty of surgery and to improve intraoperative safety drives the improvement of appropriate auxiliary equipment and technology. In fact, in order to obtain high-resolution anatomical information in LP, the application of medical imaging tools such as CT, MRI and intraoperative ultrasound has become essential.

Previously, traditional 2D CT and image reconstruction by traditional CT have been used preoperatively to assess factors that may complicate surgery, including relationships to the surrounding viscera, distribution of the vasculature, and degree of the UPJO-dependent hydronephrosis. However, due to its relatively weak ability to represent anatomical structures, including vasculature and collecting system, intuitively and stereoscopically, these traditional techniques are frequently unable to meet surgeons' demands for accurate anatomical information of targeted region. Consequently, well-established 3D image technologies based on original image data files and application of computer-aided technology have been developed to provide reliable assistance to surgeons while shortening the learning curve required for these procedures $(18,19)$.

The technology that 3D imaging relies upon has been improved through application to multiple fields such as $3 \mathrm{D}$ CT, 3D printing, 3D computed simulation surgery and 3D assisted navigations (20-22). Spiral CT (HCT) with 3D imaging, in particular, has been found to be an accurate tool to determine whether there are crossed blood vessels at the level of the UPJO. Khaira et al. (17) reported in their study that the positive predictive value for detecting crossed blood vessels with HCT is $100 \%$. The negative predictive value is $88 \%$, and the specificity and sensitivity are $100 \%$ and $91 \%$, respectively, which demonstrates that HCT with 3D imaging can serve as an effective noninvasive preoperative screening tool in the treatment of UPJO.

In the present study, we introduced a novel $3 \mathrm{D}$ visualization analysis tool to provide intuitive and omnidirectional information about the regional anatomy of the surgical area. This technology can accurately identify the position of UPJO, the structure of the surrounding vasculature and relationships with the renal parenchyma by the tinting of anatomical structures with different colors and by allowing observation with various degrees of transparency and axial rotations. In addition, this system can provide a platform for the preoperative performing of realistic surgical simulations that resemble the actual surgical procedures, which provide surgeons with unparalleled guidance and understanding leading to optimization of the surgical strategy. Preoperative planning helps the surgeon to determine the feasibility of LP in a specific situation and to design the optimal surgical plan. If the surgeon observes potential risks of intraoperative accidents during simulation process, the surgical plan may be modified and reconsidered.

According to our results, 3DIT promoted operative success, as evidenced by the facts that the 3DIT group had reduced blood loss volume and shorter time of operation and of dissection of Gerota's fascia. These improvements correlate with the understanding of the required surgical anatomy and improved anatomical orientation. In laparoscopic procedures, key information about the anatomy of the region of interest is always below the surface of the organ. In this specific case, the irregularly shaped peri-renal adipose tissue tends to blur the image, making it difficult to distinguish the anatomical structures to be targeted, including UPJ and crossing vessels. As we found in our study, in-depth surgical planning and virtual simulation can provide an improved understanding of the patient's local anatomy prior to surgery. These factors may increase the surgeon's self-confidence, which would then be expected to lead to the noted results. We also found that 3DIT can improve the efficiency of UPJ exposure. The dissection time of UPJ in 3DIT group was about one third less than that in the non-3DIT group $(\mathrm{P}=0.020)$.

The extra renal vessels that do not enter the kidney through the renal portal, called accessory renal arteries or veins, or heterotopic renal vessels (23), are of particular interest with regard to UPJO. Ectopic vessels exist in 38\% to $71 \%$ of UPJO cases and in $20 \%$ of normal kidneys, and the treatment of UPJO that is complicated by ectopic vessels is difficult and controversial. Vascular variation will affect the safety of surgery, while accidental injury to the vessels may cause bleeding. Keeping the existence and distribution of ectopic vessels in mind before operation is therefore of great significance for the choice of surgical methods, reducing intraoperative bleeding, and shortening the operation time $(7,24-26)$. The $3 \mathrm{D}$ reconstruction model based on CT can directly reflect the anatomical variation in the renal collecting system and peripheral vessels, and can help the operator to determine the location, type and direction of ectopic vessels before operation.

An ectopic artery, which maintains the blood supply to part of the kidney, is usually preserved in treatment and can be severed only when the following conditions are met: the ectopic artery clearly compresses the renal pelvis and causes 
UPJO and is thus difficult to avoid in the operation field; the ectopic artery is suspended, the tension is high. When UPJO is caused by ectopic vein compression, the non-main renal vein trunk is usually ligated and removed.

This study demonstrated the utility of 3DIT in cases involving an ectopic artery. In one case (Figure 2), the preoperative $3 \mathrm{D}$ imaging model clearly showed the position of the ectopic artery behind the ureter. The proposed operation plan was to fully free the ectopic artery and preserve the artery after UPJ, and then to perform pyeloureterostomy in front of the artery. In another case of UPJO (Figure 3), the preoperative 3D reconstruction model showed that there were annular ectopic veins in front of the renal pelvis, which were difficult to avoid in the operation area. When performing pyeloplasty, surgeons thus understood that specific attention should be paid to free the ectopic veins in the posterior segment of the UPJ. Thus, these cases demonstrate that clinicians can make accurate preoperative planning for different vascular variations of UPJO patients based on preoperative $3 \mathrm{D}$ reconstruction model information, and make targeted positioning, ligation and disconnection of vessels during operation, so as to avoid unnecessary vascular damage and to improve the accuracy and efficiency of operation.

In the group that underwent traditional CT examination, although CT images were able to provide detailed information, it might be difficult for the surgeon to observe the number and distribution of ectopic vessels and the adjacent relationship with the UPJ clearly and intuitively. The difficulty in processing this vital information may have led to the observed increases in the difficulty of the operation and the chances of bleeding due to the presence of vascular variation, as well as longer operation times and times of Gerota's fascia dissection the non-3DIT group compared with the 3DIT group.

Previous reports have similarly suggested that $3 \mathrm{D}$ reconstructions of preoperative imaging led to better surgical performance in urological laparoscopic surgery. Porpiglia et al. (27) showed that, according to nephrology scores, $3 \mathrm{D}$ visual models are more accurate than $2 \mathrm{D}$ standard imaging in assessing the surgical complexity of kidney masses, because they can provide urologists with a better understanding of tumor location, relationships and depth with intrarenal structures, leading to a better likelihood of consensus among evaluators and simplifying preoperative planning. Wake et al. (28) emphasized the workflow and new application of $3 \mathrm{D}$ printing and AR kidney model, which can provide preoperative guidance for robotic partial nephrectomy, and the insights gained by advanced visualization techniques can influence the decision-making of surgical planning. Porpiglia et al. (29) identified a hyperaccuracy $3 \mathrm{D}$ virtual navigation technique that can faithfully represent the renal artery vascular system and can thus maximize the effect of selective clamping in robot assisted partial nephrectomy. Rabah et al. (30) highlighted the use of $3 \mathrm{D}$ reconstructed images with helical CT angiography, which provides valuable preoperative information for UPJO patients who plan to undergo surgical intervention. Their research shows that this technique can be used to select the appropriate surgical treatment according to the anatomy of the cross vessels, so as to obtain a higher success rate. Khaira et al. (17) agreed that 3D reconstruction image is an accurate predictor of the presence of cross vessels in UPJO.

While these results demonstrate the utility of $3 \mathrm{D}$ technology in assisting renal surgeons, 3DIT has seldom been used in upper urinary tract reconstruction. As far as we know, ours was the first study of LP for UPJO comparing 3 DIT with the use of traditional 2D images. Some limitations of this study, though, must be recognized. First, the lack of randomization and the retrospective basis of the evaluation are the main limiting factors of our research, as they may lead to selection bias. In addition, clinical data were collected from a small patient group in a single center, and a larger population study involving multi-institution prospective, randomized and controlled trials is needed to improve confidence in the results of the research. Moreover, long-term follow-up should be conducted in further studies to better assess outcomes. Finally, all operations were performed by an experienced surgeon in our study, and inexperienced beginners should be further evaluated. 3DIT is still not available routinely in clinical practice and requires the support and cooperation of biomedical engineers.

Despite these limitations, this study shows that the assessment of the anatomical complexity of UPJO depends strictly on the imaging tools used to evaluate it. The emergence of 3DIT opens a new chapter for the surgical planning of LP, makes the classification of cross vessels more accurate and reliable, and ultimately improves the indications of surgery.

\section{Conclusions}

In summary, this study shows that in assessing the collection system, 3DIT is more accurate than standard 2D CT 
images, because they it provides urologists with a better understanding of the location and relationship of the UPJ to the structures surrounding the kidney, so as to better reach an assessment, simplify preoperative planning, and promote dissection around the UPJ during LP, leading to more accurate and safe dissections of UPJ.

\section{Acknowledgments}

The authors thank the entire staff of the Department of Urology, Peking University First Hospital.

Funding: None.

\section{Footnote}

Reporting Checklist: The authors have completed the STROBE reporting checklist. Available at https://dx.doi. org/10.21037/tau-21-590

Data Sharing Statement: Available at https://dx.doi. org/10.21037/tau-21-590

Conflicts of Interest: All authors have completed the ICMJE uniform disclosure form (available at https://dx.doi. org/10.21037/tau-21-590). XL serves as an unpaid editorial board member of Translational Andrology and Urology. The other authors have no conflicts of interest to declare.

Ethical Statement: The authors are accountable for all aspects of the work in ensuring that questions related to the accuracy or integrity of any part of the work are appropriately investigated and resolved. The study was conducted in accordance with the Declaration of Helsinki (as revised in 2013). The study was approved by the Ethics Committee of Peking University First Hospital (approval number: 2020-283) and individual consent for this retrospective analysis was waived.

Open Access Statement: This is an Open Access article distributed in accordance with the Creative Commons Attribution-NonCommercial-NoDerivs 4.0 International License (CC BY-NC-ND 4.0), which permits the noncommercial replication and distribution of the article with the strict proviso that no changes or edits are made and the original work is properly cited (including links to both the formal publication through the relevant DOI and the license). See: https://creativecommons.org/licenses/by-nc-nd/4.0/.

\section{References}

1. Hashim H, Woodhouse CRJ. Ureteropelvic junction obstruction. European Urology Supplements 2012;11:25-32.

2. Khan F, Ahmed K, Lee N, et al. Management of ureteropelvic junction obstruction in adults. Nat Rev Urol 2014;11:629-38.

3. Wang P, Xia D, Ma Q, et al. Retroperitoneal laparoscopic management of ureteropelvic junction obstruction in patients with horseshoe kidney. Urology 2014;84:1351-4.

4. Rubenwolf P, Ziesel C, Beetz R, et al. Presentation, Management and Long-Term Outcome of Ureteropelvic Junction Obstruction in Duplex Kidneys. J Urol 2015;194:427-32.

5. Osbun N, Ellison JS, Lendvay TS. Robot-Assisted Laparoscopic Excision of Ureteral and Ureteropelvic Junction Fibroepithelial Polyps in Children. J Endourol 2016;30:896-900.

6. Sampaio FJ, Favorito LA. Ureteropelvic junction stenosis: vascular anatomical background for endopyelotomy. J Urol 1993;150:1787-91.

7. Zeltser IS, Liu JB, Bagley DH. The incidence of crossing vessels in patients with normal ureteropelvic junction examined with endoluminal ultrasound. J Urol 2004;172:2304-7.

8. Stern JM, Park S, Anderson JK, et al. Functional assessment of crossing vessels as etiology of ureteropelvic junction obstruction. Urology 2007;69:1022-4.

9. Schuessler WW, Grune MT, Tecuanhuey LV, et al. Laparoscopic dismembered pyeloplasty. J Urol 1993;150:1795-9.

10. Gettman MT, Neururer R, Bartsch G, et al. AndersonHynes dismembered pyeloplasty performed using the da Vinci robotic system. Urology 2002;60:509-13.

11. Jarrett TW, Chan DY, Charambura TC, et al. Laparoscopic pyeloplasty: the first 100 cases. J Urol 2002;167:1253-6.

12. Piaggio LA, Franc-Guimond J, Noh PH, et al. Transperitoneal laparoscopic pyeloplasty for primary repair of ureteropelvic junction obstruction in infants and children: comparison with open surgery. J Urol 2007;178:1579-83.

13. Rouvière $\mathrm{O}$, Lyonnet $\mathrm{D}$, Berger $\mathrm{P}$, et al. Ureteropelvic junction obstruction: use of helical CT for preoperative assessment--comparison with intraarterial angiography. Radiology 1999;213:668-73.

14. Yang K, Yao L, Li X, et al. A modified suture technique for 
transperitoneal laparoscopic dismembered pyeloplasty of pelviureteric junction obstruction. Urology 2015;85:263-7.

15. Fang CH, Tao HS, Yang J, et al. Impact of threedimensional reconstruction technique in the operation planning of centrally located hepatocellular carcinoma. J Am Coll Surg 2015;220:28-37.

16. Chen RN, Moore RG, Kavoussi LR. Laparoscopic pyeloplasty. Indications, technique, and long-term outcome. Urol Clin North Am 1998;25:323-30.

17. Khaira HS, Platt JF, Cohan RH, et al. Helical computed tomography for identification of crossing vessels in ureteropelvic junction obstruction-comparison with operative findings. Urology 2003;62:35-9.

18. Teber D, Guven S, Simpfendörfer T, et al. Augmented reality: a new tool to improve surgical accuracy during laparoscopic partial nephrectomy? Preliminary in vitro and in vivo results. Eur Urol 2009;56:332-8.

19. Lasser MS, Doscher M, Keehn A, et al. Virtual surgical planning: a novel aid to robot-assisted laparoscopic partial nephrectomy. J Endourol 2012;26:1372-9.

20. Shimizu F, Uehara $M$, Oatari $M$, et al. Three-dimensional visualization of the human face using DICOM data and its application to facial contouring surgery using free anterolateral thigh flap transfer. J Plast Reconstr Aesthet Surg 2016;69:e1-4.

21. Souzaki R, Kinoshita Y, Ieiri S, et al. Three-dimensional liver model based on preoperative CT images as a tool to assist in surgical planning for hepatoblastoma in a child. Pediatr Surg Int 2015;31:593-6.

22. Cicione A, Autorino R, Laguna MP, et al. Threedimensional Technology Facilitates Surgical Performance of Novice Laparoscopy Surgeons: A Quantitative Assessment on a Porcine Kidney Model. Urology

Cite this article as: Zhu W, Xiong $\mathrm{S}, \mathrm{Xu} \mathrm{C}$, Zhu Z, Li Z, Zhang L, Guan H, Huang Y, Zhang P, Zhu H, Lin J, Li X, Zhou L. Initial experiences with preoperative three-dimensional image reconstruction technology in laparoscopic pyeloplasty for ureteropelvic junction obstruction. Transl Androl Urol 2021;10(11):4142-4151. doi: 10.21037/tau-21-590
2015;85:1252-6.

23. Rydberg J, Kopecky KK, Tann M, et al. Evaluation of prospective living renal donors for laparoscopic nephrectomy with multisection CT: the marriage of minimally invasive imaging with minimally invasive surgery. Radiographics 2001;21 Spec No:S223-36.

24. Boylu U, Oommen M, Lee BR, et al. Ureteropelvic junction obstruction secondary to crossing vesselsto transpose or not? The robotic experience. J Urol 2009;181:1751-5.

25. Breda A, Finelli A, Janetschek G, et al. Complications of laparoscopic surgery for renal masses: prevention, management, and comparison with the open experience. Eur Urol 2009;55:836-50.

26. Ukimura O. Image-guided surgery in minimally invasive urology. Curr Opin Urol 2010;20:136-40.

27. Porpiglia F, Amparore D, Checcucci E, et al. Threedimensional virtual imaging of renal tumours: a new tool to improve the accuracy of nephrometry scores. BJU Int 2019;124:945-54.

28. Wake N, Bjurlin MA, Rostami P, et al. Three-dimensional Printing and Augmented Reality: Enhanced Precision for Robotic Assisted Partial Nephrectomy. Urology 2018;116:227-8.

29. Porpiglia F, Fiori C, Checcucci E, et al. Hyperaccuracy Three-dimensional Reconstruction Is Able to Maximize the Efficacy of Selective Clamping During Robot-assisted Partial Nephrectomy for Complex Renal Masses. Eur Urol 2018;74:651-60.

30. Rabah D, Soderdahl DW, McAdams PD, et al. Ureteropelvic junction obstruction: does CT angiography allow better selection of therapeutic modalities and better patient outcome? J Endourol 2004;18:427-30. 